\title{
TEMPERATURA ACUMULADA DIARIA (ADD) Y SECUENCIA DE DESARTICULACIÓN EN LA RECONSTRUCCIÓN DEL INTERVALO POSTMORTEM (IPM). CONTRIBUCIÓN A LA TAFONOMÍA FORENSE
}

\author{
ACCUMULATED DEGREE DAYS (ADD) AND SEQUENCE OF \\ DISARTICULATION IN THE RECONSTRUCTION OF POSTMORTEM \\ INTERVAL (PMI). A CONTRIBUTION TO FORENSIC TAPHONOMY
}

Atilio Nasti ${ }^{1 *}$
${ }^{\prime}$ Escuela de Gendarmería Nacional (ESCUGEN). Área Criminalística y Estudios Forenses. La Matanza, Argentina

PALABRAS CLAVE tafonomía forense; temperatura acumulada; secuencia de desarticulación; intervalo postmortem

RESUMEN En este artículo se evalúan la relación entre la secuencia de desarticulación de un ejemplar de Equus caballus) y el intervalo postmortem (IPM). Para tal efecto, se propone la ecuación de regresión lineal de Megyesi, Nawrocki y Haskell (2005) en la que se utilizan los datos de temperatura local y estado de conservación de Equus caballus para obtener el ADD $\left({ }^{\circ} \mathrm{C}\right)$ acumulados por día) y así inferir el IPM. La utilización de datos comparativos forenses sobre restos animales y humanos obtenidos en otros contextos ambientales nos permite concluir que, pese al isomorfismo existente entre diferentes especies, los facto- res locales, principalmente la temperatura, producirían en Equus caballus un retraso significativo en la desarticulación durante los primeros siete meses postmortem. Aunque este estudio es preliminar, y son necesarias más investigaciones a nivel regional y microrregional, se concluye que entre el IPM esperado, derivado de la ecuación de Megyesi, y el IPM real no habría diferencias significativas, por lo que se espera que este tipo de mediciones puedan ser aplicadas en contextos discretos en donde las variables ambientales sean identificadas y cuantificadas. Rev Arg Antrop Biol 21(1), 2019. doi:10.17139/raab.2019.0021.01.07

KEY WORDS forensic taphonomy; accumulated temperature; sequence of disarticulation; postmortem interval human remains

ABSTRACT This paper presents the results on the relationship between the disarticulation sequence of Equus caballus) and the postmortem interval (PMI). For this purpose, we proposed the linear regression equation developed by Megyesi, Nawrocki and Haskell (2005) in which the local temperature data and state of conservation of Equus caballus are used to obtain the ADD (accumulated degree $\left({ }^{\circ} \mathrm{C}\right)$ days) and infer the PMI. The use of forensic comparative data on animal and human remains recovered in other environmental contexts allows us to conclude that, despite the isomorphism between different species, local factors,

El estudio y rol de la tafonomía ha crecido en forma sostenida durante los últimos años entre las disciplinas forenses (Haglund y Sorg, 1997; Haglund, Reay y Swindler , 1989; Pokines y Symes, 2013; Ubelaker, 1997;). Si bien como disciplina fue desarrollada originalmente para interpretar el registro fósil en paleontología (Efremov, 1940), ha contribuido enormemente a la comprensión de los fenómenos que afectan a los restos humanos en los contextos forenses y además a la estimación del Intervalo Post-Mortem (IPM) (Henssge y Madea, 2007; Ubelaker, 1997;). Debido a esto, un creciente interés por la tafonomía dentro del área forense ha surgido para crear métodos de aná- mainly temperature, may produce a significant delay in the sequence of disarticulation of Equus caballus during the first seven months postmortem. Although this study is preliminary and further research at regional and microregional level is required, it can be concluded that there are no significant differences between the PMI expected, derived from Megyesi's equation, and the real PMI, so this type of measurements can be expected to be applied in discrete contexts in which environmental variables are identified and quantified. Rev Arg Antrop Biol 21(1), 2019. doi:10.17139/raab.2019.0021.01.07

lisis más precisos y obtener datos más confiables (Haglund y Sorg, 1997; Pokines y Symes, 2013).

Financiamiento: Plan Estratégico Institucional 2016-2020DDNG 77/16. Secretaría de Posgrado, Investigación y Educación Permanente. Instituto Universitario de la Gendarmería Nacional, IUGNA.

*Correspondencia a: Atilio Nasti. Escuela de Gendarmería Nacional (ESCUGEN). Calle El Quebracho S/N. 1778 Ciudad Evita, La Matanza. Buenos Aires. Argentina. E-mail: anasti@iugna.edu.ar

Recibido 16 Agosto 2017; aceptado 22 Mayo 2018

doi:10.17139/raab.2019.0021.01.07 
En este sentido, las investigaciones sobre las variables medioambientales locales y sus efectos sobre los restos cadavéricos, son de gran interés debido al potencial que ofrecen para comprender los fenómenos implicados en el proceso de descomposición (Micozzi, 1986). Muchos de los factores críticos que afectan a los restos de diferentes vertebrados han sido publicados, produciendo avances significativos desde la década de 1980 (Campobasso, Vella e Introna, 2001; Galloway, 1997; Galloway, Birkby, Jones, Henry y Parks, 1989; Haglund, 1997a, b y c; Mendonça, Cocilovo y Pereda, 1984; Suckling, 2011; Ubelaker, 1997; entre otros). Sin embargo, son algunas características medioambientales discretas (temperatura, humedad, precipitación, acceso a carroñeros, etc.) las que han demostrado ser esenciales para comprender los efectos que operan sobre un cadáver (Brooks, 2016; Campobasso et al., 2001; Haynes, 1982; Taylor, 2011; Toots, 1965). Por lo demás, el tema sigue siendo complejo tanto para los científicos como para los investigadores forenses ya que inferir el intervalo post-mortem (IPM), sobre todo en ejemplares esqueletizados, implica tomar en consideración la interacción de dichas variables, lo que contribuye frecuentemente a la multiplicidad de conclusiones sobre un mismo fenómeno (Haglund y Sorg, 1997; Sledzik, 1998; Megyesi et al., 2005; Moffatt et al., 2016).

Debido a la escasez de estudios especializados en nuestro país, el objetivo de este trabajo es presentar los resultados sobre la secuencia de desarticulación de un cadáver de Equus caballus (Wilson, Reeder y Dee, 2005) a lo largo de un año (marzo 2016-abril 2017), en una zona medanosa de la costa bonaerense y su relación con el intervalo postmortem (IPM). El índice de articulación de un esqueleto de vertebrado y la secuencia de desarticulación como un proceso continuo, puede utilizarse para la construcción de modelos que den cuenta de los modos en que los huesos se disgregan anatómicamente, inferir el agente que lo produce y también el tiempo en que dicho proceso ocurre (Haynes, 1982; Hill, 1979a y b; Nasti, 2000; Reed, 2001).

La hipótesis que pretendemos testear es la existencia de una correlación positiva entre la temperatura, el índice de articulación, la secuencia de desarticulación y el IPM. Con el objeto de apoyar dicha relación, se propone aplicar la ecuación de regresión lineal de Megyesi et al., (2005) para obtener el ADD (accumulated degree day: grados ${ }^{\circ} \mathrm{C}$ acumulados por día) y así inferir el IPM.

\section{MATERIAL Y MÉTODOS}

La zona de estudio (Fig.1), se encuentra en la localidad de Mar de Las Pampas (3715'14'S; 56 56'53'O) en el borde oriental de la Pampa Húmeda (Del Río et al., 2010). El área se caracteriza por una elevada heterogeneidad de hábitats naturales como la franja de playa arenosa, sistemas de dunas activas fijas y semifijas, áreas forestadas artificiales y la llanura pampeana (Cabrera 1971; Del Río, et al., 2010). La franja de playa costera presenta dos unidades geomorfológicas diferentes, la playa y el médano, siendo la primera de forma rectilínea con un ancho que oscila entre 50 y $150 \mathrm{~m}$ mientras que la segunda presenta pendientes suaves, formadas por arenas finas a medianas (Bertola, Isla, Cortizo y Turno Orellano, 2002). La temperatura media anual es de $14,6^{\circ} \mathrm{C}$ con un promedio de precipitación de $885 \mathrm{~mm}$ y con una evapotranspiración potencial de $727 \mathrm{~mm}$. Las precipitaciones se distribuyen durante todo el año, aunque aumentan en los meses de Diciembre y Enero, disminuyendo durante el invierno (Tabla 1) (Del Río et al., 2010; Parker y Violante, 1989).

La observación se centró en el cadáver de un ejemplar juvenil (alrededor de un año y medio de edad) de Equus caballus, (identificado como MP1) localizado en marzo de 2016 sobre el borde de un médano a 60 metros de la línea de pleamar (37¹8'47.15”S; 570'26.05”O) (Fig.2). Se constató que MP1 murió a fines de marzo del mismo año, a partir del cual se relevó el proceso de esqueletización, el índice de articulación y la secuencia de desarticulación.

El relevamiento de MP1 se inició en el mes de Mayo y fue repetido en octubre, noviembre y diciembre de 2016 y enero, marzo y abril de 2017. Para evaluar el estado de articulación de un esqueleto se utilizó el método propuesto por Hill (1979a) modificado, donde se toma en cuenta el cociente entre los huesos desarticulados en relación al número total de articulaciones, $(f=$ número total de huesos del esqueleto / huesos desarticulados). La secuencia de desarticulación se comparó con los resultados obteni- 


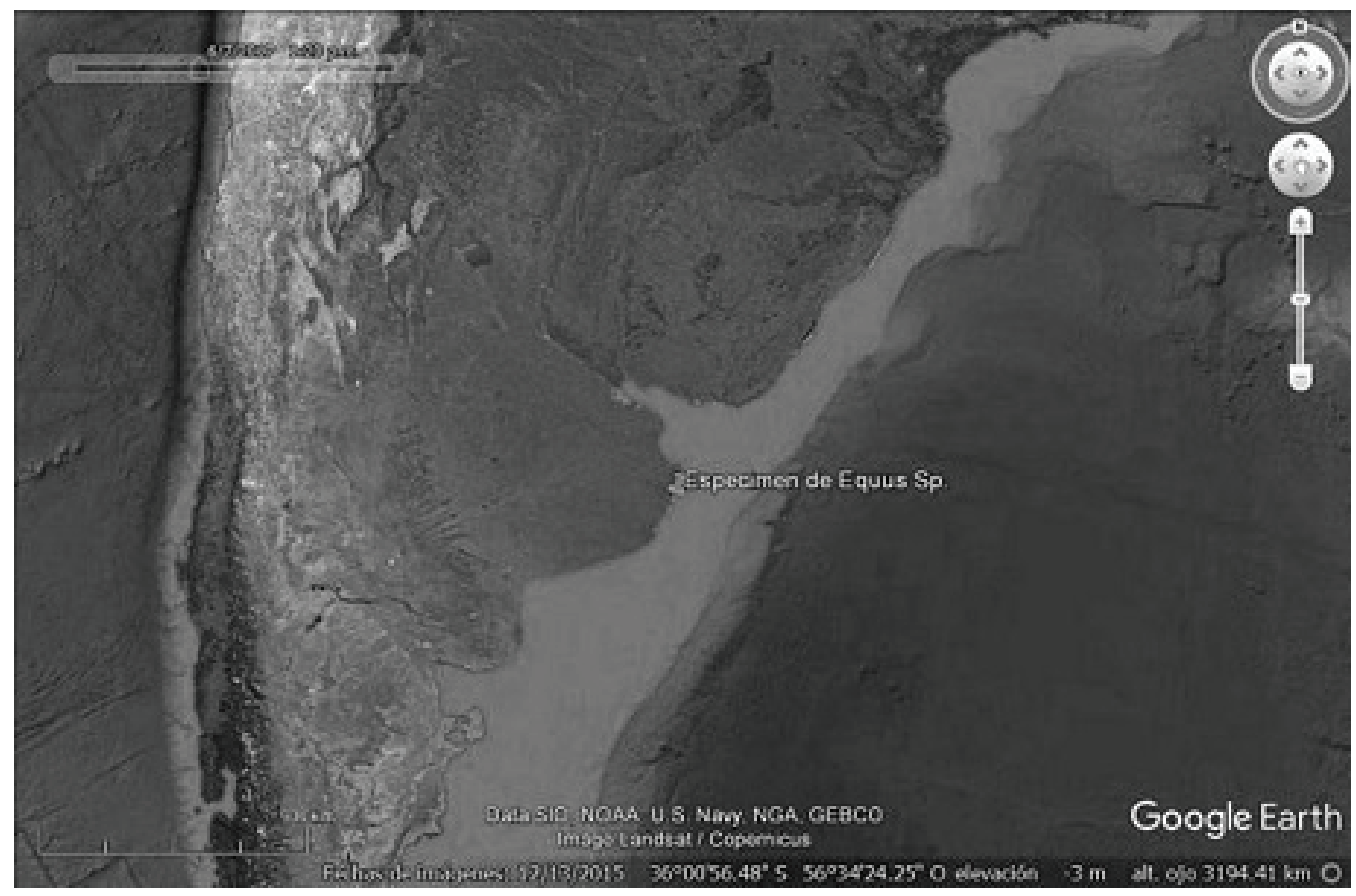

Fig. 1. Área donde se localizó el ejemplar de Equus caballus (MP1). Lat. 37 18’ 47.15” S. Long. 570’26.05”O.

TABLA 1. Datos climáticos para Mar de Las Pampas, año 2016

\begin{tabular}{ccccccccccccc}
\hline $\begin{array}{c}\text { Temperatura } \\
\left({ }^{\circ} \mathbf{C}\right)\end{array}$ & Enero & Febrero & Marzo & Abril & Mayo & Junio & Julio & Agosto Septiembre Octubre Noviembre Diciembre \\
\hline media & 20.8 & 20.7 & 18.9 & 14.8 & 11.7 & 8.9 & 8.3 & 9.2 & 10.6 & 13.4 & 16.8 & 18.9 \\
mínima & 15.1 & 15 & 13.9 & 10.0 & 7.1 & 4.8 & 4.2 & 4.6 & 6.2 & 8.8 & 11.4 & 13.2 \\
máxima & 26.6 & 26.4 & 24 & 19.6 & 16.3 & 13.1 & 12.5 & 13.9 & 15.1 & 18 & 22.2 & 24.6 \\
\hline
\end{tabular}

dos para restos de otros vertebrados (Cameron y Oxenham, 2012; Hill, 1979a; Nasti, 2000) y humanos (Haglund et al., 1989).

Con el objeto de reconstruir el IPM, se utilizó la ecuación de regresión de Megyesi et al. (2005), y para ello se obtuvieron datos de registros de temperatura (máxima y mínima diaria) en grados centígrados a lo largo del año 2016 y 2017 proporcionada por la estación meteorológica automática 876630 del aeropuerto de Villa Gesell (SAZV, Lat. 37.23, Long. -57.03, Alt. 7) (http://www.smn.gov.ar/dpd/pron5d-calendario. php). Los datos numéricos fueron tratados a través del paquete estadístico XLStat 0.15 . Para estimar el IPM con la ecuación de regresión lineal de Megyesi et al. (2005), es necesario primero reconocer los cambios en las diferentes etapas del proceso de descomposición y ser capaces de cuantificar dichas diferencias. Hay muchos sistemas de categorizar las señales visuales de la descomposición (Grassberger et al., 2004; Haglund y Sorg, 1997; Megyesi et al., 2005; Payne, 1965). El método de cuantificación que se utiliza en este trabajo se basa en un puntaje corporal total o total body score (TBS) que ha sido ensayado y publicado en los últimos años para determinar el intervalo post-mortem (IPM) en cadáveres humanos y porcinos (Galloway et al., 1989; Megyesi et al., 2005; Vass, 2011).

El TBS es una medida que cuantifica el 


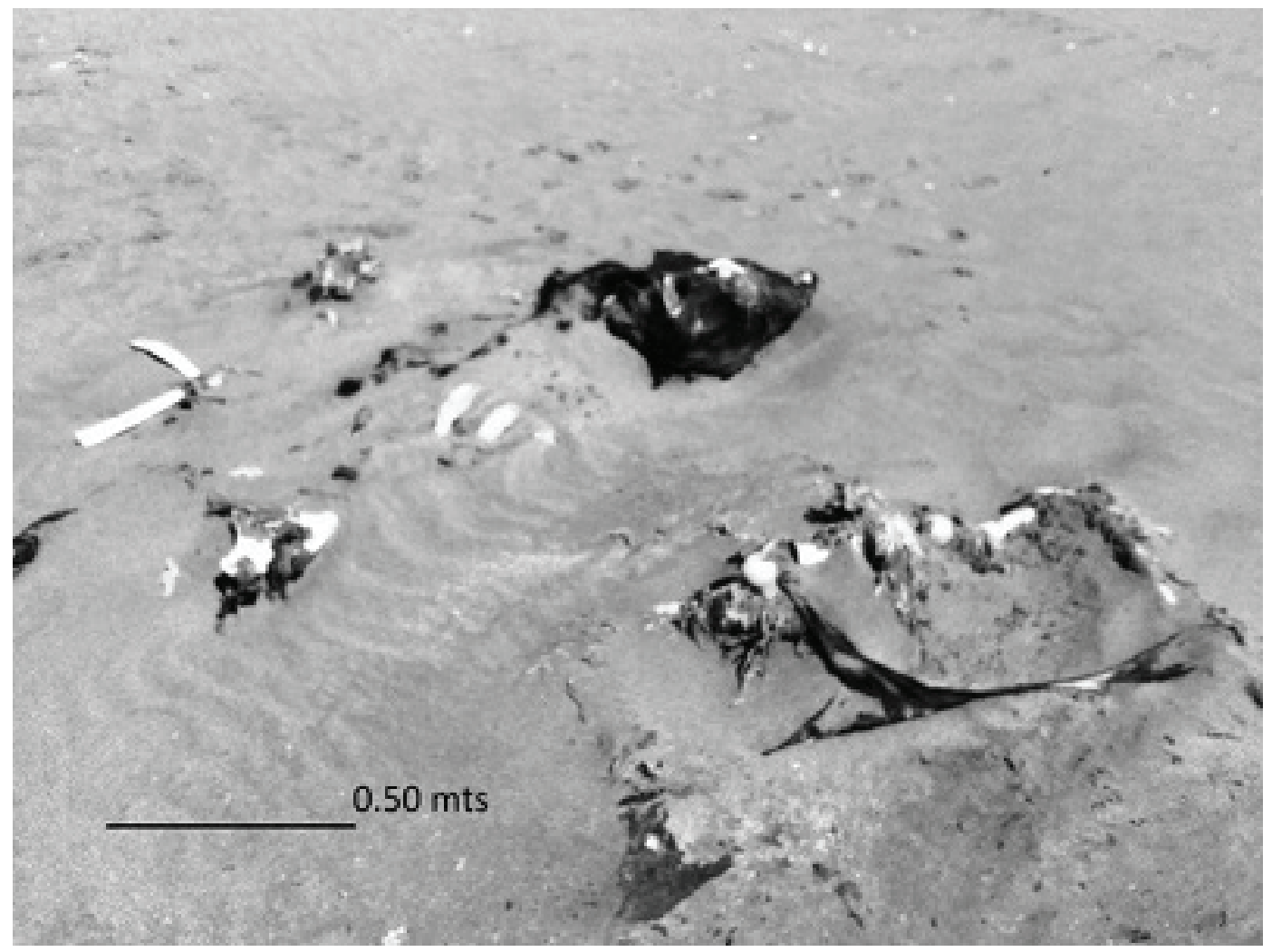

Fig. 2. Ejemplar de Equus caballus (MP1).

cambio macroscópico de la descomposición, dividiendo el cuerpo en tres regiones (cabeza, cuello, tronco y extremidades) y utilizando una escala para asignar un número a cada fase de descomposición. Estos números parciales se suman en una cifra final para generar un resultado que cuantifica la descomposición (Galloway et al., 1989; Marhoff, Fahey, Forbes y Green, 2015; Megyesi et al., 2005). Para cuantificar la variación en la tasa de descomposición, Megyesi et al. (2005) desarrollaron un método basado en la acumulación de grados centígrados por día (ADD) que permite estimar la descomposición del cuerpo a través del tiempo dependiendo de la temperatura. Utilizando la puntuación final del TBS para un cadáver, e insertando el resultado en la fórmula de regresión logarítmica:

$\log 10 \mathrm{ADD}=0.002(\mathrm{TBS} \times \mathrm{TBS})+1.81+/-388.16$

se obtiene un resultado que se interpreta como promedio de grados centígrados acumulados por día. La ADD mide la energía que se introduce en un sistema como temperatura acumulada a través del tiempo y esa misma cantidad de energía térmica se extrapola a un cuerpo, esperando la misma cantidad de reacción (descomposición). El resultado de la ecuación se extrapola regresivamente sobre la suma del promedio de temperatura diaria registrado localmente hasta obtener una fecha calendario.

Tanto Megyesi et al. (2005) como Vass (2011) presentan ecuaciones que permiten la utilización de promedios de lecturas de temperatura para el área local que se quiere evaluar. Las mismas tratan de eliminar el uso de valores residuales y así crear una fórmula de uso universal. Sin embargo, el principal problema que limita su aplicación es que las variables medioambientes tienen características locales por lo que por el momento, los ensayos experimentales dependen de ese factor para obtener resultados significativos (Moffat, Simmons y Lynch-Aird, 2016). 


\section{RESULTADOS Y DISCUSIÓN}

La Tabla 2, muestra la frecuencia de articulación obtenida para MP1, a lo largo del año en relación a la temperatura promedio. Se observa rápidamente que las lecturas de temperatura más elevadas se corresponden con una menor frecuencia de articulación (mayor desarticulación). Entre los meses de octubre y enero se registran las temperaturas promedio más elevadas $\left(20^{\circ} \mathrm{C}\right)$, disminuyendo progresivamente hacia el mes de julio con una media de $8^{\circ} \mathrm{C}$. Un aumento significativo de la media de temperatura diaria, estimularía la acción de las bacterias acelerando el proceso de putrefacción, de la desarticulación y como consecuencia de la dispersión de las diferentes partes anatómicas de MP1.

En la Figura 3, se puede observar la relación entre la temperatura promedio y la frecuencia (f) de articulación. Los meses en que MP1obtuvo las frecuencias de articulación más elevadas $(f=30)$ son los meses en que se registra menor temperatura promedio $(R=-0,8894)$.

$\mathrm{Al}$ realizar una comparación entre la secuencia de desarticulación observada para MP1, la secuencia obtenida por otros autores para diferentes vertebrados (Cameron y Oxenham, 2012; Hill, 1979a; Nasti, 2000) y el modelo de Haglund et al. (1989) para restos humanos, se puede observar que no muestran una diferencia significativa $(p=0,955)$ (Tabla 3$)$. En dicha Tabla, se aprecia una secuencia de desarticulación ordinal que comienza con el cráneo, los miembros delanteros, los traseros, la columna vertebral y la pelvis en última instancia. Si se compara las líneas de tendencias logarítmicas, se podría aceptar una isomorfía entre la frecuencia de desarticulación de esqueletos de animales y el modelo para humanos (Fig. 4).

En la Tabla 4 se puede observar la aplicación de la ecuación logarítmica de Megyesi et al. (2005) sobre MP1. Durante la primera evaluación (octubre), se obtuvo un valor de 2.388 ADD, lo que equivaldría a un IPM de 210 días (7 meses), es decir a finales del mes de marzo. Los resultados no muestran diferencias significativas entre el IPM real (221 días) y la estimación en base a la ecuación logarítmica de Megyesi et al. (204 días). La misma tabla muestra un TBS de 28 puntos después de 8 meses de muerto, mientras que a partir de noviembre y hasta abril el TBS sube a 30 incrementando la desarticulación y dispersión de los restos óseos.

Los resultados de la ecuación de Megyesi muestran en MP1 una sobre estimación creciente del IPM a partir del mes de Noviembre. Como afirmaron algunos autores (Myburgh, L'Abbé, Steyn y Becker , 2013; Parsons, 2009; Schiel, 2008) cuando los restos en estado de esqueletización se acercan a los límites del TBS (35 puntos), mayor error se esperaría en los resultados del IPM derivado de la ecuación de Megyesi et al., (2005). En la Figura 5, se muestran las líneas de tendencia para el IPM real de Equus caballus, en comparación con los valores obtenidos a partir de la ecuación de Megyesi. Puede observarse que los valores de ADD tienden a aumentar exponencialmente a través del tiempo agregando diferencias significativas en los resultados.

Observaciones similares sobre los diferentes patrones de descomposición entre invierno $\mathrm{y}$ verano, fueron registradas por Myburgh et al.

TABLA 2. Secuencia de desarticulación en escala ordinal en animales según diferentes autores

\begin{tabular}{ccccccc}
\hline $\begin{array}{c}\text { Parte } \\
\text { Anatómica }\end{array}$ & $\begin{array}{c}\text { Escala } \\
\text { Ordinal }\end{array}$ & $\begin{array}{c}\text { Hill } \\
(1979)\end{array}$ & $\begin{array}{c}\text { Cameron } \text { et al. } \\
(2012)\end{array}$ & $\begin{array}{c}\text { Haglund } \\
(1989)^{*}\end{array}$ & $\begin{array}{c}\text { Nasti } \\
(2000)\end{array}$ & $\begin{array}{c}\text { Nasti MP1 } \\
(2016)\end{array}$ \\
\hline Cráneo & 1 & 2 & 1 & 1 & 2 & 2 \\
M. Delanteros & 2 & 3 & 2 & 3 & 3 & 1 \\
Costilla & 3 & 1 & 3 & 2 & 1 & 3 \\
M. Traseros & 4 & 4 & 4 & 4 & 4 & 4 \\
Columna & 5 & 5 & 6 & 5 & 5 & 6 \\
Pelvis & 6 & 6 & 5 & 6 & 6 & 5 \\
\hline
\end{tabular}

M.:Miembros; *:Hanglund presenta resultados sobre restos humanos. 


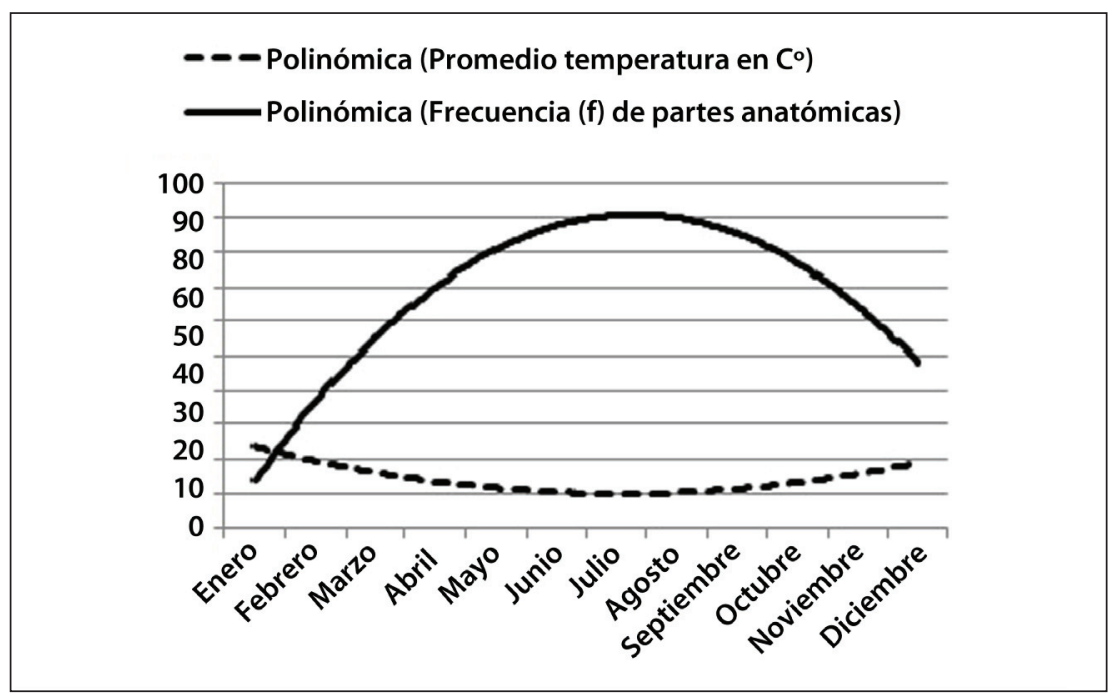

Fig. 3. Diferencia de trayectoria polinómica entre temperatura $\left({ }^{\circ} \mathrm{C}\right)$ y frecuencia $(f)$ de articulación a lo largo del año 2016.

TABLA 3. Promedio de temperatura $\left({ }^{\circ} \mathrm{C}\right)$ y frecuencia (f) de articulacion para MP1

\begin{tabular}{cccc}
\hline Año & Mes & Promedio ${ }^{\circ} \mathrm{C}$ & Frecuencia $(f)$ \\
\hline \multirow{8}{*}{2016} & Abril & 14.8 & 30.0 \\
& Mayo & 11.7 & 30.0 \\
& Junio & 8.9 & 30.0 \\
& Julio & 8.3 & 30.0 \\
& Agosto & 9.2 & 30.0 \\
& Septiembre & 10.6 & 30.0 \\
& Octubre & 13.4 & 30.0 \\
& Noviembre & 16.8 & 22.5 \\
& Diciembre & 18.9 & 10.5 \\
\hline \multirow{3}{*}{2017} & Enero & 20.8 & 8.5 \\
& Febrero & 20.7 & 7.5 \\
& Marzo & 18.9 & 7.0 \\
\hline
\end{tabular}

$f:$ número total de huesos del esqueleto/huesos desarticulados

(2013), siendo el verano donde la acción microbiana es más intensa y se alcanzan los valores más altos de descomposición y mayor rango en el resultado del TBS.

Aunque no fue tomada como una variable crítica en la desarticulación de Equus caballus, no podemos descartar la presencia de alteración antrópica. En esta región la circulación antrópica disminuye sensiblemente desde el mes de abril hasta finales de noviembre, para luego incrementarse a partir de diciembre. La dispersión de partes esqueléticas ya desarticuladas aumenta en este periodo y podría explicarse como consecuencia de este fenómeno. En este sentido, se han observado la presencia de huellas de pisoteo y otras escoriaciones que adquieren características significativas en aquellos huesos ya desarticulados y que se dispersan hacia el final de la secuencia de desarticulación (noviembre y diciembre) (Nasti, 2018).

Es interesante notar que según el modelo de Vass (2011), para inferir el IPM, la cifra de 1285 ADD sería el momento en donde se detendría el fenómeno de putrefacción y se entraría en la fase de esqueletización. De acuerdo a los datos obtenidos para Equus caballus (MP1), 1285 ADD correspondería a fines del mes de mayo. En esta fecha el proceso de esqueletización en MP1 no habría comenzado. Según los datos climáticos locales, de marzo a octubre la temperatura y la precipitación disminuyen y la descomposición de MP1 podría decrecer, es por ese motivo que el proceso de esqueletización de MP1comenzaría en Noviembre, es decir seis meses después del modelo propuesto por Vass (2011).

La ecuación de regresión lineal de Megyesi et al. (2005) fue desarrollada para cuerpos expuestos a la intemperie, de modo que los cuerpos que no cumplieran con ese requisito, 


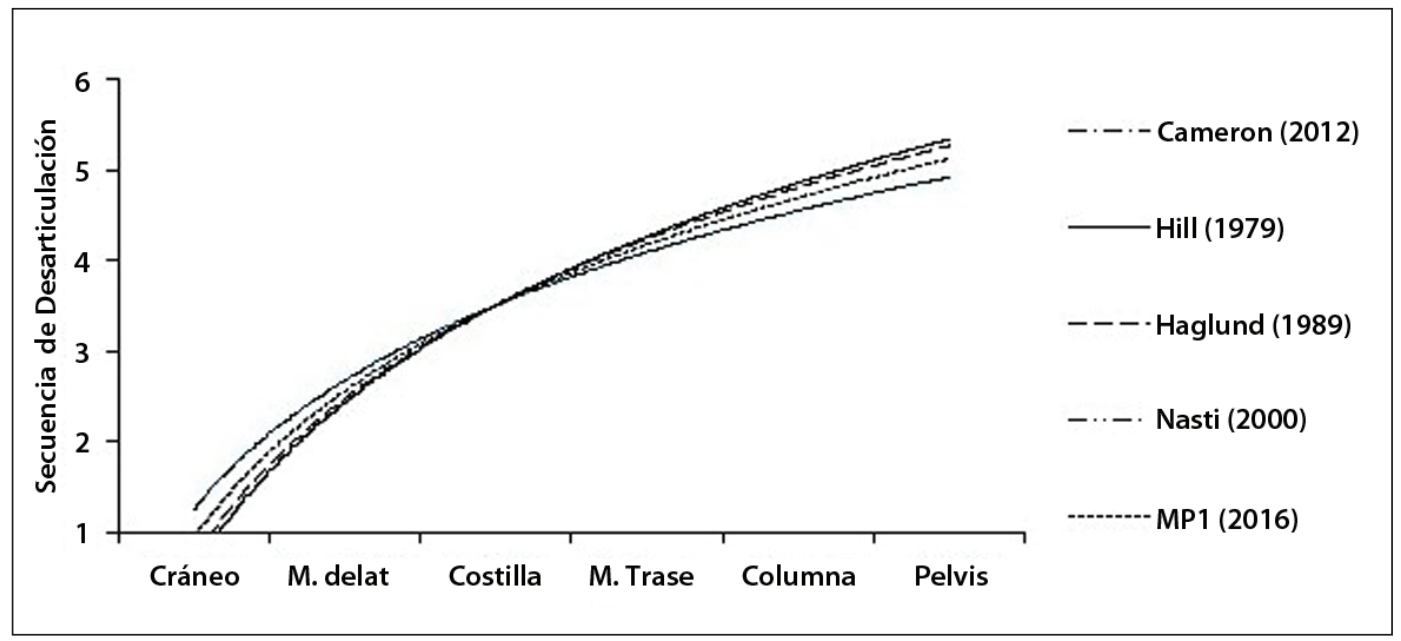

Fig. 4. Líneas de tendencia logarítmica comparativa de la secuencia de desarticulación (1 a 6) de vertebrados entre diferentes autores.

TABLA 4. Relación entre el TBS y los ADD para MP1

\begin{tabular}{ccccc}
\hline Mes & TBS & Días desde la muerte & ADD & Fecha de muerte según ADD \\
\hline Octubre & 28 & 221 & 2388 & 204 \\
Noviembre & 30 & 269 & 4073 & 304 \\
Diciembre & 32 & 284 & 7211 & 500 \\
\hline
\end{tabular}

TBS:total body score; ADD:accumulated degree day; MP1:ejemplar de Equus caballus con fecha de muerte 1/03/2016

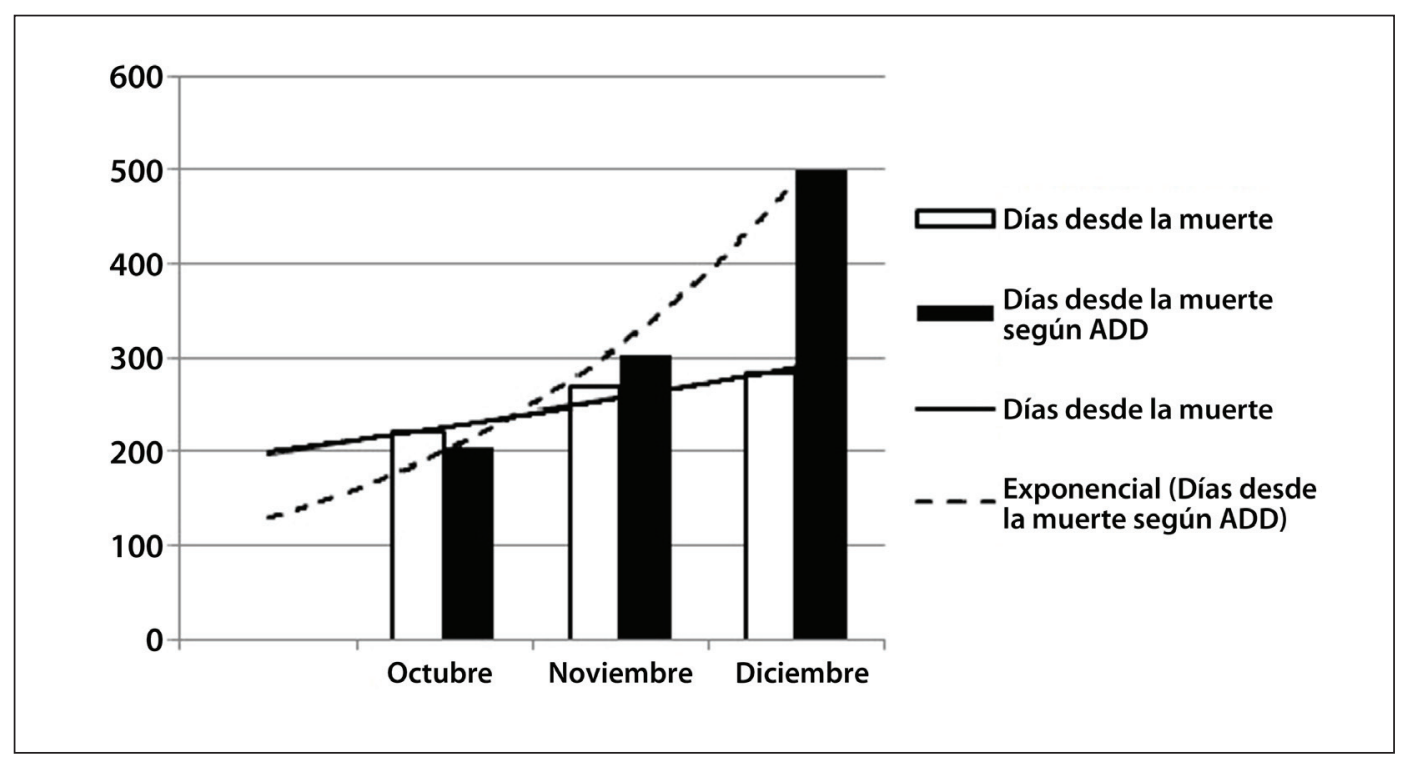

Fig. 5. Líneas de tendencia para el IPM real de Equus caballus, en comparación con los valores obtenidos a partir de la ecuación de Megyesi et al. (2005). 
al igual que la presencia de cualquier barrera donde los insectos y carroñeros puedan ser excluidos o al menos inhibidos, los resultados podrían tener importantes variaciones. Por otro lado y de modo operativo, se estima que los casos en donde se computan más de 3000ADD, el cuerpo presentaría una etapa cercana a la total esqueletización y los datos sobre el IPM podrían ser menos confiables (Moffat, et al., 2016).

Sin embargo, la aplicación de la ecuación de Megyesi et.al. (2005) sobre el ejemplar de Equus caballus, para estimar el IPM obtuvo un error de menos del $8 \%$, por lo que produce un estímulo en la búsqueda de la interdependencia entre la temperatura (ADD), la tasa de descomposición (TBS) y el IPM.

\section{CONCLUSIONES}

Si bien se han realizado importantes aportes sobre el proceso de descomposición de restos animales en diferentes ambientes utilizando metodologías alternativas (Brooks, 2016; Centeno, Maldonado y Oliva, 2002; Micozzi, 1986; Suckling, 2011; entre otros), la mayoría son de carácter cualitativo, y no son aplicables a cualquier condición geográfica y ambiental (Galloway et al., 1989; Sledzik, 1998).

Puesto que el ecosistema desempeña un papel central en el proceso de descomposición, la utilización de los modelos actuales del IPM deberían ser probados en diferentes contextos ambientales, incluso dentro de regiones geográficas específicas. Un indicador apropiado para la reconstrucción del IPM y consecuentemente la construcción de modelos tafonómicos forenses de carácter local, sería la adquisición de una base de datos adecuada sobre los procesos postdepositacionales tanto naturales como culturales que operan sobre el registro forense (Bright, 2011; Myburgh et al., 2013; Taylor 2011; Willey y Snyder, 1989).

El modelo de regresión lineal de Megyesi et al., (2005), mostró que aproximadamente el $80 \%$ de la variación en el proceso de descomposición se atribuiría a la suma de los promedios de temperatura diaria. Por lo tanto, sugiere que la descomposición debe ser modelada como dependiente de la temperatura acumulada en lugar de simplemente el tiempo transcurrido des- de la muerte. En esta dirección, los trabajos de Parsons, (2009); Schiel (2008) y Sutklin (2011), apoyarían la exactitud del método ADD/TBS de Megyesi, aunque desarrollan nuevas fórmulas adaptadas a diferentes regiones geográficas dentro de los Estados Unidos.

Este trabajo puede aportar a la tafonomía forense en dos direcciones. En primer lugar, la secuencia de desarticulación en Equus caballus es consistente con la de otros vertebrados e incluso con restos humanos (Haglund et al. 1989). Dicha secuencia es de naturaleza diacrónica y en este sentido, podría ser utilizada localmente para determinar lapsos temporales mínimos y máximos para inferir una fecha relativa del IPM. En segundo término, se obtuvo una diferencia mínima entre el IPM real y la obtenida por la ecuación de regresión lineal de Megyesi et al. (2005), (17 días) por lo que podría ser utilizada como una herramienta de naturaleza estadística para ajustar la datación del IPM.

Como todo resultado estadístico, es prioritario ajustar la ecuación en base a pruebas de carácter local y sumar otras variables de naturaleza tafonómica, sobre todo en ecosistemas donde existan datos de base registrados. La formación de una base de datos locales podría contribuir a la construcción de modelos tafonómicos forenses que podrían ser utilizados para inferir el IPM y como tal, ser relevantes en el ámbito pericial (Ubelaker, 1997).

\section{AGRADECIMIENTOS}

Agradezco los comentarios y sugerencias recibidas por parte de los evaluadores.

\section{LITERATURA CITADA}

Bertola, G., Isla, F., Cortizo, L. y Turno Orellano H. 2002. Modelo sedimentario de la barrera medanosa al norte de Villa Gesell (provincia de Buenos Aires). Asociación Argentina de Sedimentología, 9(2), 109-126. Recuperado de http://www.scielo.org.ar/pdf/raas/v9n2/v9n2a01.

Bright, L. (2011). Taphonomy signatures of animal scavenging in northern California: a forensic anthropological analysis. (Tesis de Maestría). California State University, Chico. Recuperado de https://www.csuchico.edu/bccer/ documents/Bright $\% 20$ thesis-AnimalScavenging.pdf.

Brooks J., W. (2016). Postmortem changes in animal carcasses and estimation of the postmortem interval. Veterinary $P a$ thology, 53(5), 929-940. doi:10.1177/0300985816629720.

Cabrera A. 1971. Fitogeografía de la República Argentina. Boletín de la Sociedad Argentina de Botánica, 14, 1-42.

Cameron, A. y Oxenham, M. (2012). Disarticulation sequences and scattering patterns in temperate southeas- 
tern Australia. Australian Journal of Forensic Sciences, 44:2, 197-211. doi:10.1080/00450618.2011.650206

Campobasso, C.,Vella, G. e Introna, F. (2001). Factors affecting decomposition and diptera colonization. Forensic Science International, 120, 18-27. doi:10.1016/S0379. 0738(01)00411-X

Centeno, N., Maldonado, M. y Oliva A. (2002). Seasonal patterns of arthropods occurring on sheltered and unsheltered pig carcasses in Buenos Aires province (Argentina). Forensic Science International, 126, 63-70. doi:10.1016/S0379-0738(02)00037-3

Del Río, L., Esain, M., Bocanegra, A., Zamora, A., Müller, M. y Menna, M. (2010). Caracterización y estado ambiental de Mar de Las Pampas, Partido de Villa Gesell, Provincia de Buenos Aires, República Argentina. Centro de Estudios Mar del Plata. Universidad Tecnológica Nacional. Editorial de la Universidad Tecnológica Nacional. Recuperado de http://www.edutecne.utn.edu.ar

Efremov, I. (1940). Taphonomy: new branch of palaeontology. Pan-American Geologist, 74, 81-93. http://iae. makorzh.ru/science/taph.htm

Galloway, A. (1997). The process of decomposition: a model from the Arizona-Sonoran desert. En: H. Haglund, M. Sorg (Eds). Forensic taphonomy. (pp. 139-150). Boca Raton, USA: CRC Press. doi:10.1520/JFS12680J

Galloway, A., Birkby, W., Jones, H., Henry, A. y Parks, B. (1989). Decay rates of human remains in an arid environment. Journal of Forensic Science, 34, 607-617.

Grassberger, A. y Frank, C. (2004). Initial study of arthropod succession on pig carrion in a central European urban habitat. Journal of Medical Entomology, 41(3), 511-523. doi:10.1603/0022-2585-41.3.511

Haglund, W. (1997a). Dogs and coyotes: postmortem involvement with human remains. En W. D. Haglund y M. H. Sorg (Ed.). Forensic taphonomy: the postmortem fate of human remains. (pp. 367-382). Washington, USA: CRC Press.

Haglund, W. (1997b). Rodents and human remains. En W, D Haglund y M. H. Sorg (Ed.). Forensic taphonomy: the postmortem fate of human remains. (pp. 405-414). Washington, USA: CRC Press.

Haglund W.1997c. Scattered skeletal human remains: search strategy considerations for locating missing teeth. En W. D. Haglund y M. H. Sorg (Ed.). Forensic taphonomy: the postmortem fate of human remains. (pp. 383-394). Washington, USA: CRC Press.

Haglund, W., Sorg, M. (1997). Introduction to forensic taphonomy.. En W. D. Haglund y M. H. Sorg (Ed.). Forensic taphonomy: the postmortem fate of human remains. (pp.1-9). Washington, USA: CRC Press.

Haglund, W., Reay D. T, Swindler D. R. (1989). Canid scavenging disarticulation sequences of human remains in the pacific northwest. Journal of Forensic Science, 38(1), 587-606. doi:10.1520/JFS12679J

Haynes, G. (1982). Utilization and skeletal disturbances of North American prey carcasses. Arctic, 35(2), 266-281. doi:10.14430/arctic2325

Henssge, C. y Madea, B. (2007). Estimation of the time since death. Forensic Science International,. 165, 182184.doi:10.1016/j.forsciint.2006.05.017

Hill, A. (1979a). Disarticulation and scattering of mammal skeletons. Paleobiology, 5(3), 261-274.

Hill, A. (1979b). Butchery and natural disarticulation: an investigatory technique. American Antiquity, 44(4), 739-744.

Marhoff, S., Fahey, P., Forbes, S. y Green, H. (2015). Estimating post-mortem interval using accumulated degreedays and a degree of decomposition index in Australia: a validation study. Australian Journal of Forensic Sciences, 48(1), 24-36. doi: 10.1080/00450618.2015.1021378

Megyesi M S, Nawrocki S P, Haskell N H. 2005. Using Accumulated Degree Days to Eliminate the Postmortem Interval from Decomposed Human Remains. Journal of Forensic Science. Volume 50. Pages 618 - 626. https:// repository.up.ac.za

Mendonça, O. J., Cocilovo, J. A. y Pereda, E. I. (1984). Observaciones de interés tafonómico en los restos óseos humanos del sitio "Las Lagunas" (Provincia del Neuquén). Relaciones de la Sociedad Argentina de Antropología, XVI, 235-248.

Micozzi, M.S. (1986). Experimental study of postmortem change under field conditions: effects of freezing, thawing, and mechanical injury. Journal of Forensic Science, 31(3), 953-961. doi:10.1520/JFS11103J

Moffatt, C., Simmons, T. y Lynch-Aird, J. (2016). An improved equation for TBS and ADD: establishing a reliable postmortem interval framework for casework and experimental studies. Journal of Forensic Science, 61(Suppl 1), S201-207. doi:10.1111/1556-4029.12931

Myburgh, J.N., L'Abbé, N., Steyn, M. y Becker, P. (2013). Estimating the postmortem interval (PMI) using accumulated degree-days (ADD) in a temperate region of South Africa. Forensic Science International, 229(13),165 e1-6. doi:10.1016/j.forsciint.2013.03.037

Nasti, A. (2000). Modification of vicuña carcasses in highaltitude deserts. Current Anthropology, 41(2), 279-283.

Nasti, A. (2018). An forensic comparative 3D analysis of trampling and eolian traces on modern bones. $M s$.

Parker, G. y Violante, A. (1989). Geología y geomorfología. Regiones I y II. Punta Rasa - Faro Querandí. Provincia de Buenos Aires. Convenio Consejo Federal de Inversiones y Servicio de Hidrografía Naval, Informe final. $110 \mathrm{pp}$.

Parsons, H. R. (2009). The postmortem interval: a systematic study of pig decomposition in West Central Montana. (Tesis de grado). University of Montana. Montana Graduate Student Theses, Dissertations, \& Professional Papers. 1058. https://scholarworks.umt. edu/etd/1058

Payne, J.A. (1965). A summer carrion study of the baby pig Suss crofa Linnaeus. Ecology, 46, 592-602. doi:https:// doi.org/10.2307/1934999

Pokines, J. y Symes, S. (2013). Manual of forensic taphonomy. New York, USA: CR Press.

Reed, E. H. (2001). Disarticulation of kangaroo skeletons in semi-arid Australia. Australian Journal of Zoology, 49, 615-632.

Schiel, M. (2008). Using accumulate degree days for estimating the postmortem interval: a reevaluation of Megyesi's regression formulae. (Tesis de Maestría). University of Indianapolis, Indiana.

Sledzik, P. S. (1998). Forensic taphonomy: postmortem decomposition and decay. En K.J. Reichs (Ed) Forensic osteology. (pp. 109-119). Springfield, USA: Charles C.Thomas.

Suckling, A. (2011). A longitudinal study on the outdoor human decomposition sequence in Central Texas. (Tesis Doctoral). Texas State University. Texas. Recuperado de https://digital.library.txstate.edu/bitstream/handle/10877/4072/fulltext.pdf

Taylor, L. (2011). A taphonomic study and pos-mortem interval formula comparision. (Tesis de Maestría). Cranfield University. Bedford, Inglaterra. doi:10.13140/ RG.2.1.1631.8323

Toots, H. E. (1965). Sequences of disarticulation in mammalian skeletons. Rocky Mountain Geology, 4(1), 37-39. 
Recuperado de https://pubs.geoscienceworld.org/uwyo/ $\mathrm{rmg} /$ article/4/1/37/87976/sequence-of-disarticulationin-mammalian-skeletons

Ubelaker, D. H. (1997). Taphonomic applications in forensic anthropology, taken from forensic taphonomy. En W, D Haglund y M. H. Sorg (Ed.). The postmortem fate of human remains. (pp.77-90). Washington, USA: CRC Press.

Vass, A. (2011). The elusive universal post-mortem interval formula. Forensic Science International, 204, 34-40. doi:10.1016/j.forsciint.2010.04.052

Willey, P. y Snyder LM. 1989. Canid modification of human remains: implications for time-since death estimations. Journal of Forensic Science, 34(4), 894-901. doi: $10.1520 /$ JFS12718J

Wilson, D., Reeder, A. y Dee A.F. (2005). Mammal species of the World. Baltimore, USA: Johns Hopkins University Press. 